\title{
A Comparison of Fosaprepitant and Ondansetron for Preventing Postoperative Nausea and Vomiting in Moderate to High Risk Patients: A Retrospective Database Analysis
}

\author{
Chiaki Murakami, Nami Kakuta, Katsuyoshi Kume, Yoko Sakai, Asuka Kasai, \\ Takuro Oyama, Katsuya Tanaka, and Yasuo M. Tsutsumi \\ Department of Anesthesiology, Tokushima University, 3-18-15 Kuramoto, Tokushima 770-8503, Japan \\ Correspondence should be addressed to Yasuo M. Tsutsumi; tsutsumi@tokushima-u.ac.jp
}

Received 6 September 2017; Accepted 28 November 2017; Published 19 December 2017

Academic Editor: Giovanni Mariscalco

Copyright (C) 2017 Chiaki Murakami et al. This is an open access article distributed under the Creative Commons Attribution License, which permits unrestricted use, distribution, and reproduction in any medium, provided the original work is properly cited.

\begin{abstract}
Postoperative nausea and vomiting (PONV) occur in 30-50\% of patients undergoing general anesthesia and in $70-80 \%$ of high PONV risk patients. In this study, we investigated the efficacy of fosaprepitant, a neurokinin-1 (NK1) receptor antagonist, compared to ondansetron, a selective 5-hydroxytryptamine type 3 (5-HT3) receptor antagonist, in moderate to high PONV risk patients from our previous randomized controlled trials. Patients (171 patients from 4 pooled studies) with the Apfel simplified score $\geq$ 2 and undergoing general anesthesia were randomly allocated to receive intravenous fosaprepitant $150 \mathrm{mg}$ (NK1 group, $n=82$ ) and intravenous ondansetron $4 \mathrm{mg}$ (ONS group, $n=89$ ) before induction of anesthesia. Incidence of vomiting was significantly lower in the NK1 group compared to the ONS group 0-2,0-24, and 0-48 hours after surgery ( 2 versus $17 \%, 2$ versus $28 \%$, and 2 versus $29 \%$, resp.). However, no significant differences in PONV, complete response, rescue antiemetic use, and nausea score were observed between groups 0-48 hours after surgery. In moderate to high PONV risk patients, fosaprepitant decreased the incidence of vomiting and was superior to ondansetron in preventing postoperative vomiting 0-48 hours after surgery.
\end{abstract}

\section{Introduction}

Postoperative nausea and vomiting (PONV) are distressing and serious adverse effects of anesthesia and surgery. PONV is experienced by $30-50 \%$ of patients undergoing general anesthesia and up to $70-80 \%$ of high PONV risk patients (female patients, nonsmokers, patients with history of PONV and/or motion sickness, and receiving opioids postoperatively [1-4]). In the "Consensus guidelines for the management of postoperative nausea and vomiting" [4], use of antiemetics for PONV prophylaxis is recommended in moderate to high risk patients. Additionally, in patients receiving prophylactic treatment for PONV, such as ondansetron, a selective 5-hydroxytryptamine type 3 (5-HT3) receptor antagonist, PONV, occurs in up to $30-40 \%$ of the patients during the first postoperative day $[2,5,6]$. In addition to patient distress and discomfort, PONV increases the risk of venous thrombus or pulmonary embolism, interferes with physical therapy, and prolongs hospital stay [7-9]. Therefore, more effective therapies for preventing PONV are required, particularly in high risk patients.

Fosaprepitant is a water-soluble phosphorylated prodrug of aprepitant, a highly selective neurokinin-1 (NK1) receptor antagonist, and is rapidly transformed into aprepitant when administered intravenously [10]. Aprepitant blocks NK1 receptors and demonstrates antiemetic efficacy and a long half-life, with two large trials finding aprepitant advantageous in preventing PONV, compared to ondansetron [11-13]. Fosaprepitant is used for managing chemotherapy-induced nausea and vomiting (CINV) and was reported to have superior, longer-lasting effect in preventing CINV, compared to other antiemetics [14].

Recently, we reported that fosaprepitant was more effective than ondansetron in preventing vomiting 0-24 and $0-48 \mathrm{~h}$ after surgery [15-17]. In this study, we collected data from our previous studies and evaluated the efficacy of 
fosaprepitant in preventing PONV compared to ondansetron in moderate to high PONV risk patients.

\section{Methods}

This analysis was based on data from four identically designed, double-blind, randomized, controlled studies. These studies were approved by the Human Research Ethics Committee of the University of Tokushima (Tokushima, Japan) and registered in a clinical trials database (UMIN000008621, 000012999, 000007613, and 000018532). Written informed consent was obtained from all patients, and the studies were conducted in accordance with the principles outlined in the Declaration of Helsinki.

Patients (aged 20-80 years) with the American Society of Anesthesiologists (ASA) physical status of I-III and undergoing general anesthesia were enrolled in the studies. They all had Apfel simplified score for $P O N V \geq 2$, which includes 4 factors: female sex, history of PONV and/or motion sickness, nonsmoker smoking status, and postoperative opioids use. Patients with 0-1, 2, and 3-4 factors are considered to be at low, moderate, and high risk for PONV, respectively [2]. Exclusion criteria were ASA physical status of IV, neural diseases, abnormal liver and/or renal function, and use of other antiemetics. Patient data recorded included gender, history of PONV and motion sickness, and smoking status.

Patients were randomized and allocated to groups using Quickcalcs software (Graph-Pad Inc., La Jolla, CA, USA). To ensure investigator blinding randomization schedule was generated by a statistician who was not involved in the clinical study. On the day of surgery, patients were randomized to the NK1 group, which received intravenous fosaprepitant (150 mg) and the ONS group, which received intravenous ondansetron (4 mg). Antiemetics were infused over $30 \mathrm{~min}$ by anesthesiologists before induction of anesthesia, following approved administration protocols.

Anesthesia was induced with remifentanil $0.3-0.5 \mu \mathrm{g} / \mathrm{kg} /$ $\mathrm{min}$, propofol $1-2 \mathrm{mg} / \mathrm{kg}$, and rocuronium $0.8 \mathrm{mg} / \mathrm{kg}$ to facilitate endotracheal intubation. Anesthesia was maintained with propofol (target controlled infusion: 2-4 $\mu \mathrm{g} / \mathrm{ml}$ ) or volatile anesthetics (sevoflurane $1-2 \%$ or desflurane $3-4 \%$ ) in oxygen with air mixture, remifentanil, and fentanyl. Incremental doses of rocuronium were used as necessary for muscle relaxation, which was reversed by sugammadex $2 \mathrm{mg} / \mathrm{kg}$ at the end of surgery. A rescue antiemetic (10 mg metoclopramide) and/or analgesic were administered on patient request.

The incidence of nausea and vomiting, complete response rate (no vomiting and no rescue antiemetic use), rescue antiemetic use, and severity of nausea were evaluated by blinded observers 2, 24, and 48 hours after surgery. The severity of nausea was estimated using the nausea score $(0$, absence of nausea; 1-3 mild, moderate, and severe nausea, resp.). Scores were evaluated by blinded observers, who determined the highest scores in each surveyed period and all adverse events during the first 48 hours after surgery were recorded.

Statistical analyses were performed using Statistical Package for Social Sciences (SPSS) software (version 22, SPSS
TABLE 1: Patient demographics.

\begin{tabular}{lcc}
\hline & NK1 group & ONS group \\
\hline Patients & $n=82$ & $n=89$ \\
Age, years & $57 \pm 14$ & $56 \pm 13$ \\
Height, cm & $154 \pm 8$ & $155 \pm 6$ \\
Weight, kg & $57 \pm 11$ & $56 \pm 10$ \\
ASA PS I/II/III & $23 / 58 / 1$ & $27 / 61 / 1$ \\
PONV risk factors & & \\
$\quad$ Nonsmoker $(n)$ & 80 & 88 \\
$\quad$ History of motion sickness $(n)$ & 26 & 22 \\
$\quad$ Women ( $n$ ) & 82 & 89 \\
$\quad$ Postoperative opioids, $\mu \mathrm{g}$ & $470 \pm 449$ & $443 \pm 451$ \\
Duration of anesthesia, min & $269 \pm 124$ & $280 \pm 138$ \\
Duration of surgery, min & $209 \pm 113$ & $219 \pm 129$ \\
Anesthetics; remifentanil, mg & $3.4 \pm 2.4$ & $3.2 \pm 3.2$ \\
Blood loss, ml & $321 \pm 488$ & $251 \pm 360$ \\
Fluid volume, ml & $2245 \pm$ & $2127 \pm 1101$ \\
\hline
\end{tabular}

Data are presented as means \pm SD (or range) or number of patients; NK1 group, i.v. fosaprepitant; ONS group, i.v. ondansetron; PONV, postoperative nausea and vomiting; ASA PS, American Society of Anesthesiologists physical status.

INC., Chicago, IL, USA). Data are expressed as means \pm standard deviations. $P$ values $<0.05$ were considered statistically significant without any adjustment for multiplicity of testing. $T$-test and $\chi^{2}$ test were used for analyzing patient demographics, cumulative incidence of vomiting at each time point, incidence of nausea, rescue antiemetic use, and complete response (no vomiting and no rescue) $0-2,0-24$, and $0-48$ hours after surgery. The Mann-Whitney $U$ test was used to analyze nausea score at these time points.

\section{Results}

This study included 171 female patients with the Apfel simplified score $\geq 2$ ( 82 in the NK1 group and 89 in the ONS group). No significant differences in patient demographics, risk factors for PONV, duration of anesthesia and surgery, blood loss, and fluid volume were observed between the groups (Table 1).

There was no significant difference in the percentage of patients with no vomiting and no rescue (complete response) at $0-2$ hours (78 versus $80 \%$, resp.; $P=0.852), 0-24$ hours $(71$ versus $67 \%$, resp.; $P=0.741$ ), and $0-48$ hours ( 71 versus $67 \%$, resp.; $P=0.741$ ) after surgery. However, the incidence of vomiting was significantly lower in the $\mathrm{NK1}$ group compared to the ONS group at $0-2$ hours (2 versus $17 \%$, resp.; $P=$ 0.0016 ), $0-24$ hours (2 versus $28 \%$, resp.; $P<0.0001$ ), and 0-48 hours (2 versus 29\%, resp.; $P<0.0001$ ) after surgery (Table 2). Where rates of vomiting were adjusted for use of rescue antiemetic, both groups were similar in terms of rescue antiemetic use for the $0-48 \mathrm{~h}$ interval. The rescue antiemetic was administered $10 \mathrm{mg}$ metoclopramide when the patients required postoperative rescue antiemetic. No statistically 
TABLE 2: Postoperative parameters.

\begin{tabular}{|c|c|c|c|}
\hline & NK1 group, $n=82$ & ONS group, $n=89$ & $P$ value \\
\hline \multicolumn{4}{|l|}{$0-2$ hours } \\
\hline PONV & $34(41 \%)$ & $25(28 \%)$ & 0.077 \\
\hline Complete response & $64(78 \%)$ & $71(80 \%)$ & 0.852 \\
\hline Vomiting & $2(2 \%)^{*}$ & $15(17 \%)$ & 0.0016 \\
\hline Rescue antiemetic use & 18 & 13 & 0.238 \\
\hline \multicolumn{4}{|l|}{$0-24$ hours } \\
\hline PONV & $45(55 \%)$ & $44(49 \%)$ & 0.647 \\
\hline Complete response & $58(71 \%)$ & $60(67 \%)$ & 0.741 \\
\hline Vomiting & $2(2 \%)^{*}$ & $25(28 \%)$ & $<0.0001$ \\
\hline Rescue antiemetic use & 30 & 26 & 0.331 \\
\hline \multicolumn{4}{|l|}{$0-48$ hours } \\
\hline PONV & $45(55 \%)$ & $47(53 \%)$ & 0.878 \\
\hline Complete response & $58(71 \%)$ & $60(67 \%)$ & 0.741 \\
\hline Vomiting & $2(2 \%)^{*}$ & $26(29 \%)$ & $<0.0001$ \\
\hline Rescue antiemetic use & 30 & 29 & 0.631 \\
\hline
\end{tabular}

Data are presented as number of patients (percentile); NK1 group, i.v. fosaprepitant; ONS group, i.v. ondansetron; PONV, postoperative nausea and vomiting. ${ }^{*} P<0.05$ compared to ONS group.

TABLE 3: Nausea and NRS.

\begin{tabular}{lcc}
\hline & $\begin{array}{c}\text { NK1 group, } \\
n=82\end{array}$ & $\begin{array}{c}\text { ONS group, } \\
n=89\end{array}$ \\
\hline $\begin{array}{l}\text { 0-2 hours } \\
\quad \text { Severity of nausea }(0 / 1 / 2 / 3)\end{array}$ & $48 / 15 / 10 / 9$ & $65 / 6 / 4 / 14$ \\
$\begin{array}{l}\text { 2-24 hours } \\
\quad \text { Severity of nausea }(0 / 1 / 2 / 3)\end{array}$ & $62 / 10 / 6 / 4$ & $57 / 10 / 10 / 12$ \\
$24-48$ hours & & \\
$\quad$ Severity of nausea $(0 / 1 / 2 / 3)$ & $76 / 5 / 1 / 0$ & $77 / 6 / 2 / 4$ \\
\hline
\end{tabular}

Data are presented as number of patients or median (interquartile range); NK1 group, i.v. fosaprepitant; ONS group, i.v. ondansetron; severity of nausea: $0=$ absence of nausea, $1=$ mild nausea, $2=$ moderate nausea, and $3=$ severe nausea.

significant differences in PONV, rescue antiemetic use, and nausea score were observed between the groups for any analyzed period (Tables 2 and 3 ).

\section{Discussion}

This study indicated that fosaprepitant is superior to ondansetron in preventing vomiting $0-2,0-24$, and $0-48$ hours after surgery in moderate to high PONV risk patients. No significant differences were observed between fosaprepitant and ondansetron in incidence of PONV, complete response rate, use of rescue antiemetics, and severity of nausea.

Several neurotransmitter receptor systems including dopaminergic, cholinergic, histaminergic, serotonergic (5HT2 and 5HT-3), and NK1 systems are associated with nausea and vomiting [18]. 5HT-3 receptor antagonists and NK1 receptor antagonists were shown to be effective in preventing CINV. A corticosteroid and a 5HT-3 receptor antagonist are often combined for preventing CINV. Recently, NK1 receptor antagonists, aprepitant and fosaprepitant, have become available and their use has improved the management of CINV [19]. In the 2016 update of MASCC/ESMO consensus guidelines, for prophylaxis of acute and delayed nausea and vomiting induced by chemotherapy, a combination of a 5$\mathrm{HT} 3$ receptor antagonist, dexamethasone, and a NK1 receptor antagonist is recommended [20]. NK1 receptor antagonists are expected to reduce the incidence of CINV.

NK1 receptor antagonist aprepitant was shown to be effective in preventing PONV. Diemunsch et al. [12] suggested that aprepitant was significantly superior to ondansetron in preventing vomiting. Gan et al. [11] showed that aprepitant was as effective in reducing nausea as ondansetron and more effective in preventing vomiting during the first 24 and 48 hours after surgery. Moreover, analysis of data from two clinical trials [13] indicated that aprepitant was superior to ondansetron in preventing PONV.

In this study, we used fosaprepitant, a prodrug of aprepitant. Fosaprepitant is converted to aprepitant within $30 \mathrm{~min}-$ utes of intravenous administration and blocks NK1 receptors. A dose of $115 \mathrm{mg}$ of fosaprepitant was reported equivalent to $125 \mathrm{mg}$ of aprepitant [10], with intravenous administration of $150 \mathrm{mg}$ of fosaprepitant showing the same efficacy as a 3 -day oral aprepitant treatment (125/80/80 mg) [21]. In our previous studies [15-17], we demonstrated the efficacy of fosaprepitant in preventing PONV in patients undergoing general anesthesia compared to ondansetron. This study enrolled moderate to high PONV risk patients and confirmed fosaprepitant efficacy compared to ondansetron. Improved efficacy of fosaprepitant in preventing postoperative vomiting may be attributed to its long half-life.

For moderate to high PONV risk patients, multimodal prevention approaches can reduce the risk of PONV. Consensus guidelines for managing postoperative nausea and 
vomiting [4] recommend an antiemetic combination of dexamethasone $(4 \mathrm{mg})$ and ondansetron $(4 \mathrm{mg})$. Considering the superior antiemetic effect of fosaprepitant compared to ondansetron in this study, a combination of dexamethasone and fosaprepitant (instead of ondansetron) may be more effective. Moreover, long half-life of fosaprepitant suggests that a single administration of fosaprepitant may be equivalent or more effective than a combination dexamethasoneondansetron treatment in preventing PONV. Further evaluation of clinical characteristics of fosaprepitant in combination with other antiemetics for PONV prevention is planned.

Certain limitations should be noted. Fosaprepitant has a much longer half-life than ondansetron and, consequently, longer duration of activity, which may account for improved prevention of vomiting. Additionally, as antiemetics are expected to be more effective when administered towards the end of surgery compared to during induction of anesthesia, timing of drug administration could have affected our results.

Moreover, fosaprepitant treatment is relatively expensive, costing US\$ 122.5 in Japan. However, as use of antiemetics can reduce complications associated with PONV, preventing prolonged hospital stays, total medical costs may decrease. As we did not evaluate long-term outcomes in this study, it remains unclear whether reduction of postoperative vomiting by fosaprepitant improves postoperative outcomes or provides additional benefits for patients. Further studies are required to evaluate the contribution of fosaprepitant treatment to postoperative outcomes.

\section{Conclusions}

This study demonstrated that NK1 receptor antagonist fosaprepitant was superior to 5HT-3 receptor antagonist ondansetron in preventing vomiting $0-2,0-24$, and $0-48$ hours after surgery in moderate to high PONV risk patients. However, no significant differences were observed between fosaprepitant and ondansetron in PONV incidence, complete response, use of rescue antiemetics, and nausea severity at any period analyzed.

\section{Conflicts of Interest}

The authors declare that there are no conflicts of interest regarding the publication of this article.

\section{Authors' Contributions}

C. Murakami and N. Kakuta contributed equally to this work.

\section{Acknowledgments}

This study was supported entirely by JSPS KAKENHI (Grants nos. 16K16278 and 16K10940).

\section{References}

[1] M. Koivuranta, E. Läärä, L. Snåre, and S. Alahuhta, "A survey of postoperative nausea and vomiting," Anaesthesia, vol. 52, no. 5, pp. 443-449, 1997.
[2] C. C. Apfel, E. Läärä, M. Koivuranta, C. Greim, and N. Roewer, "A simplified risk score for predicting postoperative nausea and vomiting: conclusions from cross-validations between two centers," Anesthesiology, vol. 91, no. 3, pp. 693-700, 1999.

[3] D. R. Sinclair, F. Chung, and G. Mezei, "Can postoperative nausea and vomiting be predicted?” Anesthesiology, vol. 91, no. 1, pp. 109-118, 1999.

[4] T. J. Gan, P. Diemunsch, A. S. Habib et al., "Consensus guidelines for the management of postoperative nausea and vomiting," Anesthesia \& Analgesia, vol. 118, no. 1, pp. 85-113, 2014.

[5] M. Rust and L. A. Cohen, "Single oral dose ondansetron in the prevention of postoperative nausea and emesis," Anaesthesia, vol. 49, pp. 16-23, 1994.

[6] T. J. Gan, B. Ginsberg, A. P. Grant, and P. S. A. Glass, "Double-blind, randomized comparison of ondansetron and intraoperative propofol to prevent postoperative nausea and vomiting," Anesthesiology, vol. 85, no. 5, pp. 1036-1042, 1996.

[7] F. Adam, M. Chauvin, B. Du Manoir, M. Langlois, D. I. Sessler, and D. Fletcher, "Small-dose ketamine infusion improves postoperative analgesia and rehabilitation after total knee arthroplasty," Anesthesia \& Analgesia, vol. 100, no. 2, pp. 475-480, 2005.

[8] L. D. Dorr and L. Chao, "The emotional state of the patient after total hip and knee arthroplasty," Clinical Orthopaedics and Related Research, no. 463, pp. 7-12, 2007.

[9] J. R. Backes, J. C. Bentley, J. R. Politi, and B. T. Chambers, “Dexamethasone reduces length of hospitalization and improves postoperative pain and nausea after total joint arthroplasty: a prospective, randomized controlled trial," The Journal of Arthroplasty, vol. 28, no. 8, pp. 11-17, 2013.

[10] K. C. Lasseter, J. Gambale, D. Pharm et al., "Tolerability of fosaprepitant and bioequivalency to aprepitant in healthy subjects," Clinical Pharmacology and Therapeutics, vol. 47, no. 7, pp. 834-840, 2007.

[11] T. J. Gan, C. C. Apfel, A. Kovac et al., "A randomized, doubleblind comparison of the NK1 antagonist, aprepitant, versus ondansetron for the prevention of postoperative nausea and vomiting," Anesthesia \& Analgesia, vol. 104, no. 5, pp. 1082-1089, 2007.

[12] P. Diemunsch, T. J. Gan, B. K. Philip et al., "Single-dose aprepitant vs ondansetron for the prevention of postoperative nausea and vomiting: a randomized, double-blind Phase III trial in patients undergoing open abdominal surgery," British Journal of Anaesthesia, vol. 99, no. 2, pp. 202-211, 2007.

[13] P. Diemunsch, C. Apfel, T. J. Gan et al., "Preventing postoperative nausea and vomiting: Post hoc analysis of pooled data from two randomized active-controlled trials of aprepitant," Current Medical Research and Opinion, vol. 23, no. 10, pp. 2559-2565, 2007.

[14] P. Langford and P. Chrisp, "Fosaprepitant and aprepitant: An update of the evidence for their place in the prevention of chemotherapy-induced nausea and vomiting," Core Evidence, vol. 5, pp. 77-90, 2010.

[15] Y. M. Tsutsumi, N. Kakuta, T. Soga et al., "The Effects of intravenous fosaprepitant and ondansetron for the prevention of postoperative nausea and vomiting in neurosurgery patients: A prospective, randomized, double-blinded study," BioMed Research International, vol. 2014, Article ID 307025, 2014.

[16] T. Soga, K. Kume, N. Kakuta et al., "Fosaprepitant versus ondansetron for the prevention of postoperative nausea and 
vomiting in patients who undergo gynecologic abdominal surgery with patient-controlled epidural analgesia: a prospective, randomized, double-blind study," Journal of Anesthesia \& Clinical Research, vol. 29, no. 5, pp. 696-701, 2015.

[17] N. Kakuta, K. Kume, E. Hamaguchi et al., "The effects of intravenous fosaprepitant and ondansetron in the prevention of postoperative nausea and vomiting in patients who underwent lower limb surgery: a prospective, randomized, double-blind study," Journal of Anesthesia \& Clinical Research, vol. 29, no. 6, pp. 836-841, 2015.

[18] P. Diemunsch, G. P. Joshi, and J.-F. Brichant, "Neurokinin-1 receptor antagonists in the prevention of postoperative nausea and vomiting," British Journal of Anaesthesia, vol. 103, no. 1, pp. 7-13, 2009.

[19] M. Aapro, A. Carides, B. L. Rapoport, H.-J. Schmoll, L. Zhang, and D. Warr, "Aprepitant and fosaprepitant: A 10-year review of efficacy and safety," The Oncologist, vol. 20, no. 4, pp. 450-458, 2015.

[20] L. H. Einhorn, B. Rapoport, R. M. Navari, J. Herrstedt, and M. J. Brames, "2016 updated MASCC/ESMO consensus recommendations: prevention of nausea and vomiting following multipleday chemotherapy, high-dose chemotherapy, and breakthrough nausea and vomiting," Supportive Care in Cancer, vol. 25, no. 1, pp. 303-308, 2017.

[21] S. Grunberg, D. Chua, A. Maru et al., "Single-dose fosaprepitant for the prevention of chemotherapy-induced nausea and vomiting associated with cisplatin therapy: Randomized, doubleblind study protocol-EASE," Journal of Clinical Oncology, vol. 29, no. 11, pp. 1495-1501, 2011. 


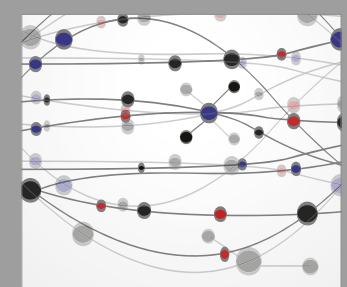

The Scientific World Journal
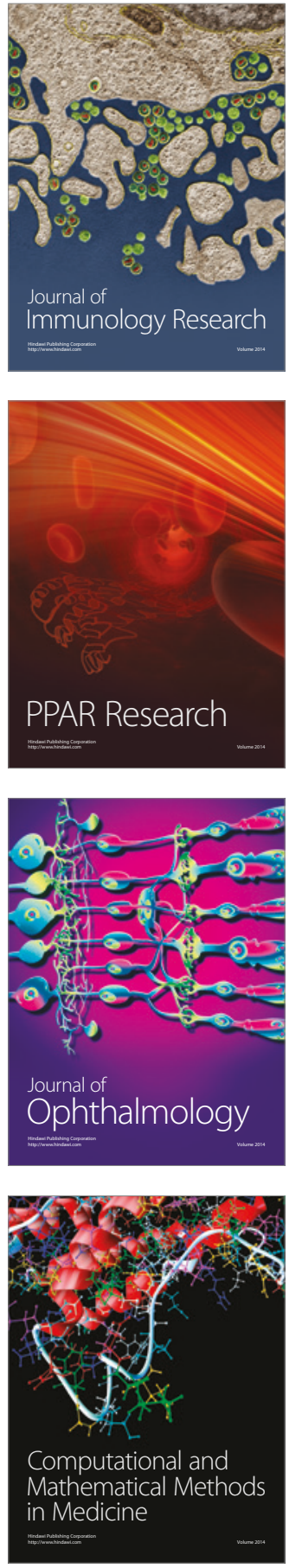

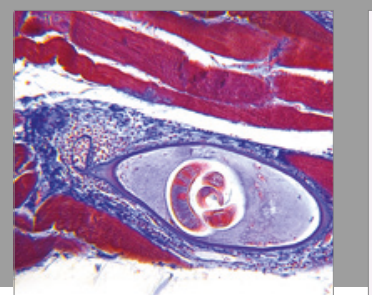

Gastroenterology Research and Practice
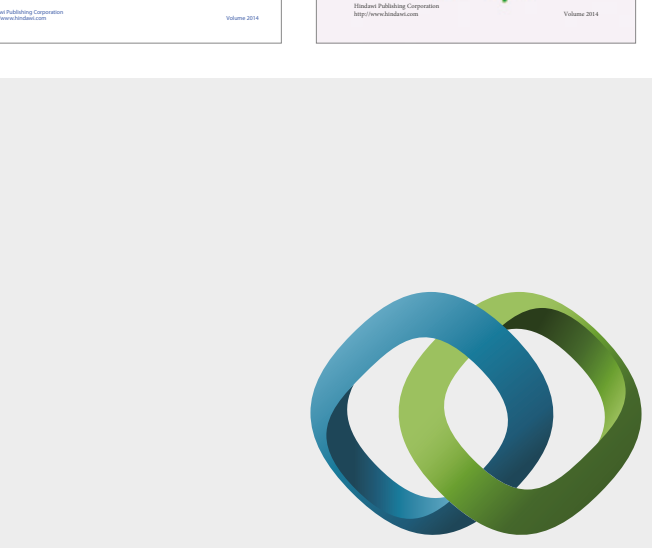

\section{Hindawi}

Submit your manuscripts at

https://www.hindawi.com
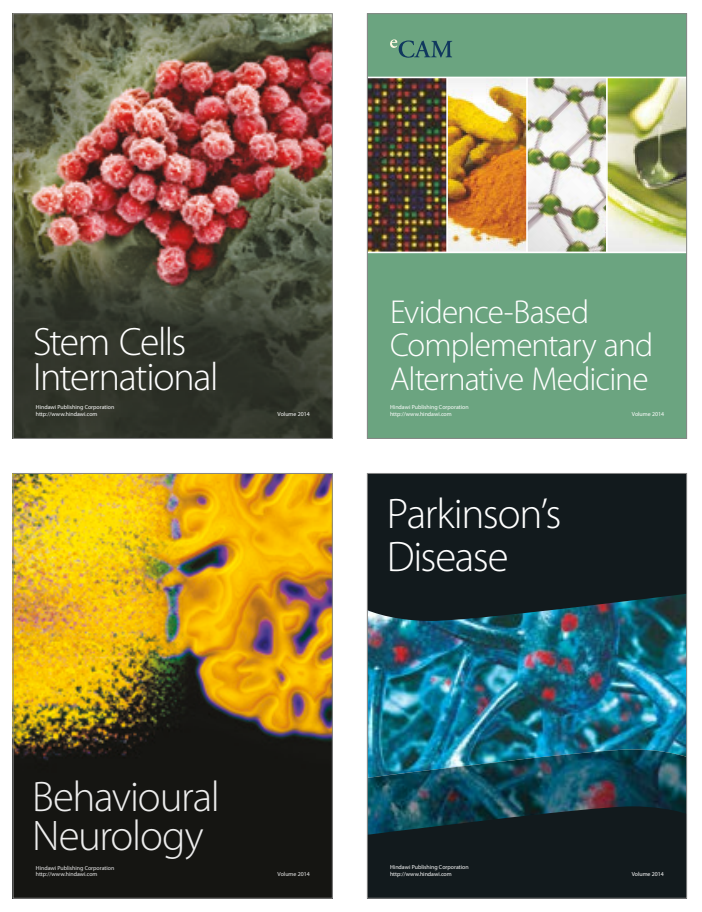
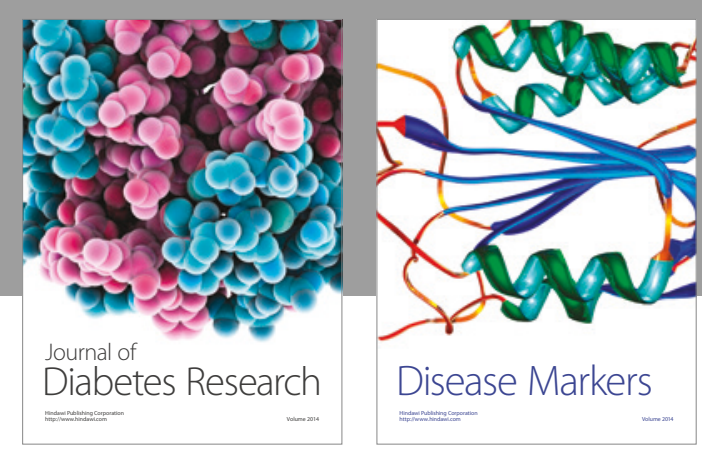

Disease Markers
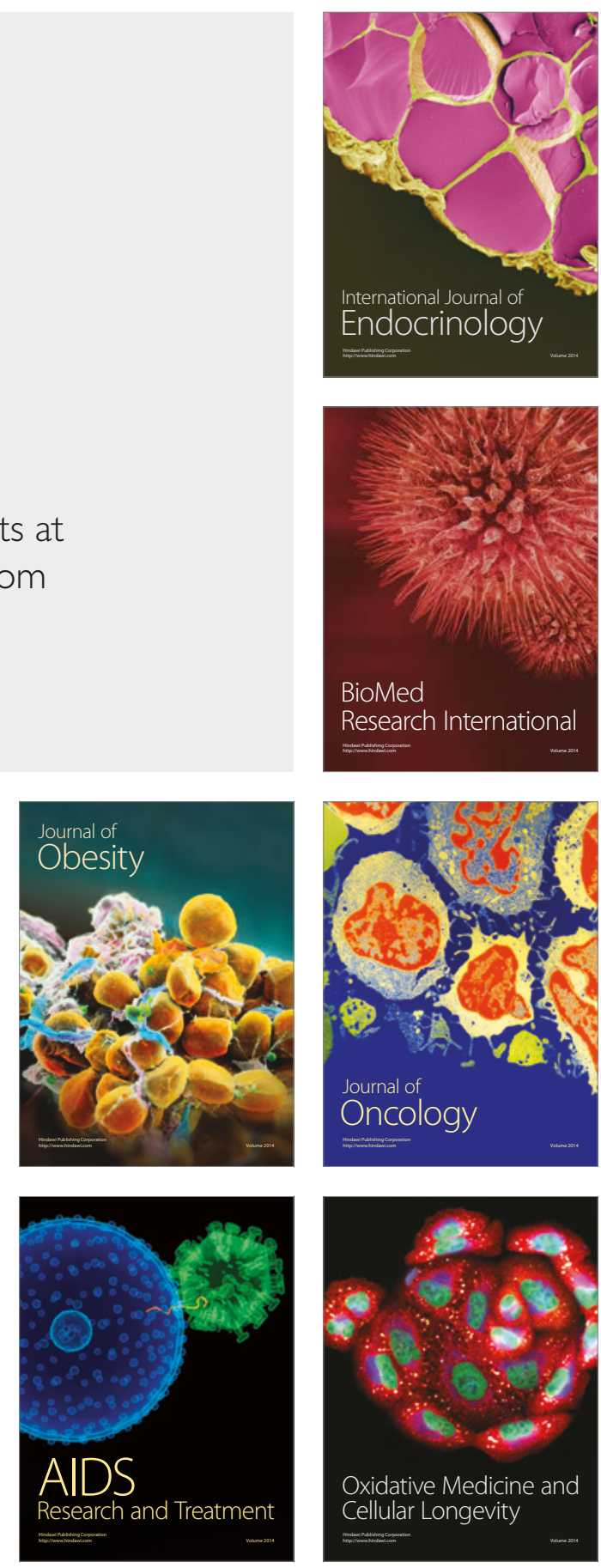